\title{
PROGRAMA DE DESARROLLO ACADÉMICO PARA ALUMNOS DE PRIMER AÑO
}

\author{
Bernardo Gómez B. * \\ Lidia Osorio* \\ Paula Domínguez* \\ María Gabriela Silva* \\ Dagoberto Navea* \\ Marta Monárdez* \\ Angélica Silva*
}




\section{RESUMEN}

Un número creciente de alumnos se está incorporando a la educación superior en Chile. Destacan entre ellos los jóvenes provenientes de sectores socioeconómicos más modestos, los que, en condiciones de notoria precariedad, deben competir con sus compañeros de sectores socioeconómicos acomodados. Ello atenta en contra de sus posibilidades de finalizar sus estudios. Estos jóvenes representan, sin embargo, la capacidad del sistema de promover la movilidad social, por lo que las instituciones de educación superior deben prestarles especial atención y apoyo. La identificación de estos jóvenes mediante indicadores de vulnerabilidad como el que se propone, además de la generación de acciones específicas para su protección, incorpora en la Universidad el sentido de la equidad, y favoreciendo su retención, impulsa la movilidad social y el desarrollo del país.

\section{ABSTRACT}

An increasing number of students is going on to higher education in Chile. Outstanding among these are young people from less well-to-do economic sectors who-at a notorious disadvantagemust compete with their fellow students from wealthier income levels. This undermines their possibilities of completing their studies. However, these young people represent the system's capability to promote social mobility. Consequently, higher education establishments need to provide them with special attention and support. Identifying these young people by means of vulnerability indicators such as the one proposed-in addition to carrying out specific actions for their protection-brings in to the University the idea of equality and favors their retention, promotes social mobility and the development of the country. 


\section{PROGRAMA DE DESARROLLO ACADÉMICO PARA ALUMNOS DE PRIMER AÑO}

\section{INTRODUCCIÓN}

Una clara tendencia del sistema educacional chileno en las últimas décadas ha sido el incremento del número de alumnos que se matriculan cada año en la educación superior. Según cifras del Ministerio de Educación, la matrícula ha crecido muy por sobre el crecimiento poblacional, lo que habla no sólo del aumento de la oferta educacional en Chile, sino también de un fenómeno a nuestro juicio no menor: que un creciente número de alumnos provenientes de familias de niveles socioeconómicos bajos o medio bajos está accediendo a la educación superior en el país.

Este fenómeno, que apunta no sólo al logro de una mayor equidad en el acceso a la educación superior, sino, además, a la creciente capacidad de retención de la educación media en Chile, significa por otro lado un importante desafío para las instituciones de educación superior del país, del que pareciera no hemos tomado aún clara conciencia.

En el concierto latinoamericano, nuestro país ha mantenido cifras altas de movilidad social, esto es: las posibilidades que tiene un chileno pobre de que sus hijos puedan ascender en la escala social son notoriamente superiores a las de nuestros países vecinos. Este fenómeno, además de estar íntimamente ligado a la educación, por ser éste el factor de mayor peso específico en el logro de la movilidad social de un país, es también un indicador clave en sus posibilidades de desarrollo, porque toda acción que promueva la movilidad social es, de por sí, una potente acción en pro del desarrollo de ese colectivo.

Hay abundante evidencia de que los talentos de las personas se distribuyen en forma bastante democrática en una comunidad, de 
modo que, tanto la inteligencia como ciertas habilidades de los individuos, si bien son rasgos que aparecen ligados a la herencia, no demuestran que ésta tenga directa consonancia con el nivel socioeconómico de las familias de origen. De tal forma, afortunadamente, los hijos de los ricos no son más inteligentes ni talentosos que los hijos de los pobres, por lo que la educación, como vehículo de desarrollo, potenciación y canalización de habilidades y talentos de las personas, resulta ser de crucial importancia para el desarrollo de una comunidad.

En las últimas décadas nos ha preocupado también la creciente brecha registrada en la calidad de la educación media entre el sistema público y el privado, brecha que parece explicarse mejor por un desarrollo de éste último, más que por un deterioro del primero. Sin embargo, en regiones es visible que un número importante de alumnos provenientes del sistema de educación pública, tanto de los establecimientos de administración municipal como de los colegios privados subvencionados por el Estado (esto es, que imparte educación "gratuita" para el apoderado), logra terminar con éxito su educación media y cumplen satisfactoriamente con los requisitos para el ingreso a la educación superior.

En nuestro sistema educacional, especialmente en la educación superior, existe una natural orientación hacia la excelencia académica, esto es, el lucimiento de los mejores. De hecho, un criterio para la distribución del crédito fiscal a las instituciones sigue siendo la preferencia que los mejores puntajes en las pruebas de ingreso al sistema muestran por una determinada casa de estudios. No es menos cierto, por otra parte, que los alumnos provenientes de sectores socioeconómicos más altos cuentan en general con más apoyos para cursar sus carreras, no sólo respecto de la satisfacción de sus necesidades básicas, sino también de la disposición de condiciones y materiales de estudio. ¿Qué ocurre entonces con aquellos jóvenes provenientes de familias de sectores más modestos? Ellos tienen que enfrentar los mismos niveles de exigencia, compitiendo de igual a igual con sus compañeros más privilegiados económicamente, pero claramente en condiciones mucho más precarias, muchas veces lejos de sus familias, sin las circunstancias más adecuadas en cuanto a vivienda y, a veces, incluso con déficit de alimentación, habida cuenta 
que todo esto ocurre en una etapa de la vida de suyo compleja y vulnerable, cual es la adolescencia. Nos parece de especial importancia, entonces, equilibrar la necesaria orientación a la excelencia académica de nuestra Universidad, con un sano sentido de equidad, esto es, dar a cada cual lo que requiere y, en ese entendido, nos ha parecido de toda justicia poner en evidencia la necesidad de discriminar positivamente entre nuestros alumnos, a aquellos con mayores vulnerabilidades, para focalizar sobre ellos acciones de apoyos específicos. De esta forma buscamos favorecer su permanencia en la Universidad, su éxito académico y por consiguiente, promover su movilidad social y a través de ellos, el desarrollo de la colectividad.

El creciente número de jóvenes que ingresan a nuestras universidades provenientes de familias de niveles socioeconómicos más bajos representan la expectativa de progreso de sus familias, se transforman en modelos entre sus pares y otorgan a las instituciones de educación superior la oportunidad de ejercitar y poner en juego toda su capacidad formativa, en términos de crear con ellos profesionales capaces de abrir nuevos caminos y favorecer el cambio y el progreso de nuestro país. El ingreso de estos alumnos a nuestras universidades es entonces una oportunidad única de dar un salto cualitativo, que nos permita no sólo un recambio de profesionales calificados, sino ampliar además sus bases del desarrollo.

La enseñanza superior es la mejor medida para marcar el cambio que en la movilidad social de los individuos representa la educación, como oportunidad de mejores niveles de empleo, así como porque el acceso más equitativo a la información y a la cultura permite la creación de una masa crítica, mejor dotada para generar las transformaciones que los tiempos van exigiendo a nuestra sociedad.

Se trata, por tanto, de lograr que la integración efectiva de estos alumnos a la Universidad se transforme en una experiencia de crecimiento y evolución que marque positivamente sus vidas. Por consiguiente, el desafío para nuestras universidades no se cifra sólo en impartir conocimiento con excelencia o en ser centros formadores de alto nivel, sino además, en hacer todo ello con equidad. Esto implica identificar a nuestros alumnos más vulnerables y orientar hacia ellos acciones de protección específica, pues como hemos señalado, son 
ellos los que representan el verdadero desafío formativo. Resulta obvio recordar que lo fácil está al alcance de cualquiera y que, en cambio, lo difícil marca una diferencia evolutiva. Nuestras universidades no sólo deben impartir ciertas materias y esperar que los mejores las asimilen como es deseable, sino que deben saber reconocer las características personales en los jóvenes y adaptar los contenidos a entregarles, con el objeto de potenciar su rendimiento y optimizar su formación. En ningún caso proponemos el facilismo, sino la flexibilidad curricular, los programas diferenciados de aprendizaje y programas de apoyo específico, capaces de hacer de la equidad una necesaria y complementaria orientación de nuestro sistema de educación superior.

\section{EVALUACIÓN DE LA VULNERABILIDAD ACADÉMICA}

Nos ha interesado estudiar las condiciones de vulnerabilidad que presentan los alumnos ingresados al primer año de pregrado de la Universidad Arturo Prat en Iquique. Definimos vulnerabilidad, como la posibilidad de sufrir daño y, en este caso específico, referimos el daño a la reprobación de ramos y abandono de los estudios superiores. De entre las muchas formas en que podríamos acercarnos a este problema hemos elegido, por razones prácticas, la de estudiar la información disponible respecto de factores socioeconómicos y académicos que pueden asociarse a este riesgo.

Todas las universidades cuentan con bases de datos de los alumnos que postulan a los diversos beneficios que otorga el sistema, desde el crédito fiscal para el financiamiento de los estudios, hasta las diferentes becas que el sistema o cada casa de estudios ofrece a sus alumnos. En la Universidad Arturo Prat un poco más de la mitad de los matriculados en el primer año postula a alguno de estos beneficios; por consiguiente, se dispone de una base de datos con una cantidad de información objetivamente completa respecto de las condiciones socioeconómicas de los postulantes.

Nos preguntamos $i$ cuáles de todos estos factores pueden asociarse con la posibilidad de reprobación o abandono de estudios en estos alumnos? Para ello se cruzó la información disponible con los rendimientos en años anteriores, con lo que se pudo construir un 
índice de vulnerabilidad que considera siete variables, con diferente ponderación, para la evaluación de la vulnerabilidad académica. Este índice no predice rendimiento según nuestro estudio, pero sí permite focalizar de un modo suficientemente razonable las acciones de protección específicas que se programan con los alumnos.

Las variables seleccionadas, su puntaje y ponderación se presentan en la siguiente tabla.

\section{Tabla $\mathrm{N}^{\circ} 1$ :}

Índice de vulnerabilidad académica: para cada variable existe un puntaje según la categoría de cada alumno; a su vez, a cada variable se le asigna una ponderación, según su relevancia como localizador de las intervenciones.

\begin{tabular}{|c|c|c|}
\hline VARIABLE & PUNTAJE & PONDERACIÓN \\
\hline Ingreso per cápita del grupo familiar & $\begin{array}{l}>\$ 100.000=0 \\
\$ 50.000 \text { a } \$ 100.000=1 \\
<\$ 50.000=2\end{array}$ & $25 \%$ \\
\hline $\begin{array}{l}\text { Tipo de colegio de egreso } \\
\text { de enseñanza media }\end{array}$ & $\begin{array}{l}\text { Particular pagado =0 } \\
\text { Part. subvencionado =1 } \\
\text { Municipalizado = } 2\end{array}$ & $15 \%$ \\
\hline Escolaridad de los padres & $\begin{array}{l}\text { Sin estudios }=2 \\
\text { Básicos incompletos }=1.9 \\
\text { Básicos completos }=1.8 \\
\text { Medios incompletos }=1.7 \\
\text { Medios completos }=1.0 \\
\text { Técnicos incompletos }=0.7 \\
\text { Técnicos completos }=0.5 \\
\text { Profesionales completos }=0\end{array}$ & $20 \%$ \\
\hline $\begin{array}{l}\text { Residencia del grupo familiar } \\
\text { (distancia del alumno) }\end{array}$ & $\begin{array}{l}<50 \mathrm{Km} .=0 \\
50 \mathrm{Km} . \mathrm{a} 500 \mathrm{Km} .=1 \\
>500 \mathrm{Km} .=2\end{array}$ & $15 \%$ \\
\hline $\begin{array}{l}\text { Preferencia en su postulación respecto } \\
\text { de matrícula }\end{array}$ & $\begin{array}{l}1^{\text {a }} \text { opción }=0 \\
2^{\text {a }} \text { opción }=1 \\
3^{\text {a }} \text { opción }=2\end{array}$ & $5 \%$ \\
\hline Puntaje PAA. & $\begin{array}{l}>600 \text { pts. }=0 \\
\text { de } 500 \text { a } 600 \text { pts. }=1 \\
<500 \text { pts. }=2\end{array}$ & $10 \%$ \\
\hline Carga horaria semestral de la carrera & $\begin{array}{l}<25 \text { hrs. }=0 \\
\text { de } 25 \text { a } 30 \text { hrs. }=1 \\
>30 \text { hrs. }=2\end{array}$ & $10 \%$ \\
\hline
\end{tabular}


Este índice se ha confeccionado con el propósito fundamental de identificar a aquellos alumnos que presentan mayores vulnerabilidades en cuanto a reprobar ramos y, con ello, prolongar y encarecer sus estudios o abandonar sus carreras. Las variables escogidas no representan factores de riesgo en sí mismas, sino más bien, condiciones socioeconómicas y académicas, que dificultan o pueden dificultar el rendimiento y la integración a la vida universitaria. Por cierto, dicho índice puede ser perfeccionado, correlacionando de forma dinámica las variables anotadas -y otras- con el rendimiento; además se pueden realizar estudios específicos en alumnos que reprueban o abandonan sus estudios, para identificar factores de riesgo asociado.

Nuestro interés es continuar profundizando en el tema, toda vez que, como hemos dicho, con el propósito de favorecer la movilidad social resulta de vital importancia la capacidad de retención que la Universidad logra con sus alumnos, especialmente con los de sectores socioeconómicos más desprovistos.

Con el indicador presentado, se estudió la población de alumnos que postulan a beneficios en nuestra Universidad, con los resultados que se presentan en el siguiente gráfico:

\section{Gráfico $\mathrm{N}^{\circ} 1$ :}

Evaluación de vulnerabilidad académica; alumnos de primer año, Universidad Arturo Prat, 2002.

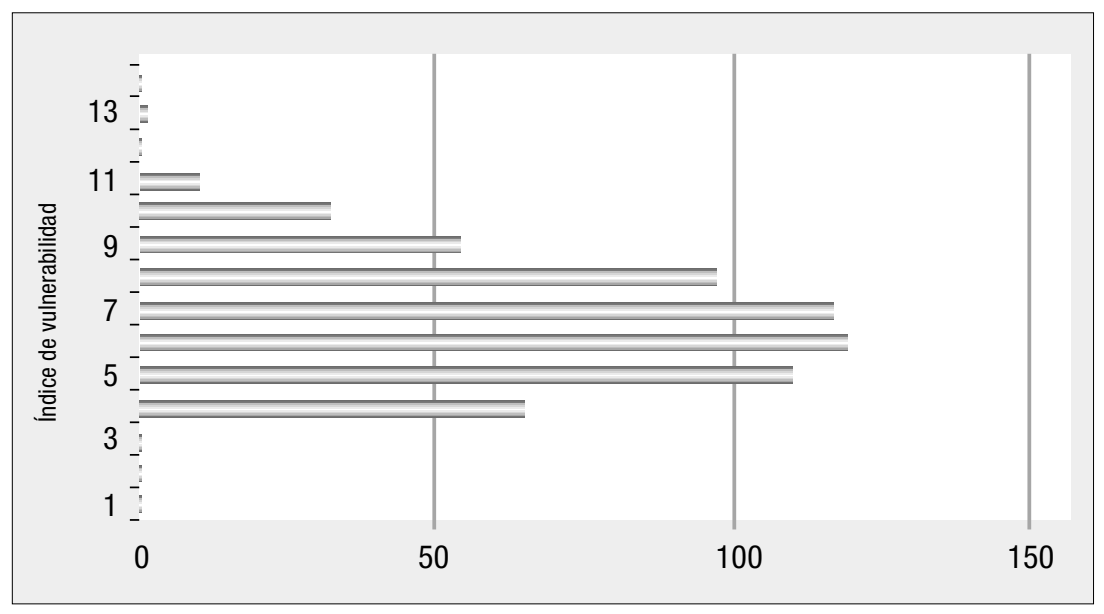


En la ciudad de Iquique, el año 2002, se matricularon 1.158 alumnos en el primer año de las diferentes carreras que imparte la Universidad Arturo Prat; de estos alumnos, 611 (52.7\%) postularon a algún beneficio. Al estudiar el índice de vulnerabilidad académica en ese grupo, se encontró una distribución normal del indicador: con un valor máximo posible de 14 puntos, el valor más frecuente o moda se situó en 6 puntos. Si se considera que a mayor valor mayor vulnerabilidad, 311 alumnos de los estudiados (50.9\%) presentan valores de vulnerabilidad por sobre el valor modal, lo que representa una distribución del indicador en estos alumnos con una leve carga hacia condiciones de mayor vulnerabilidad, expresado en valores más altos del indicador.

\section{Gráfico $\mathrm{N}^{\circ} 2$ :}

Se muestra el diseño del programa, con un grupo de alumnos incorporados al programa y un grupo control, pareado por igual valor en su índice de vulnerabilidad, con el propósito de comparar en la evaluación, sus rendimientos y nivel de satisfacción e integración a sus estudios.

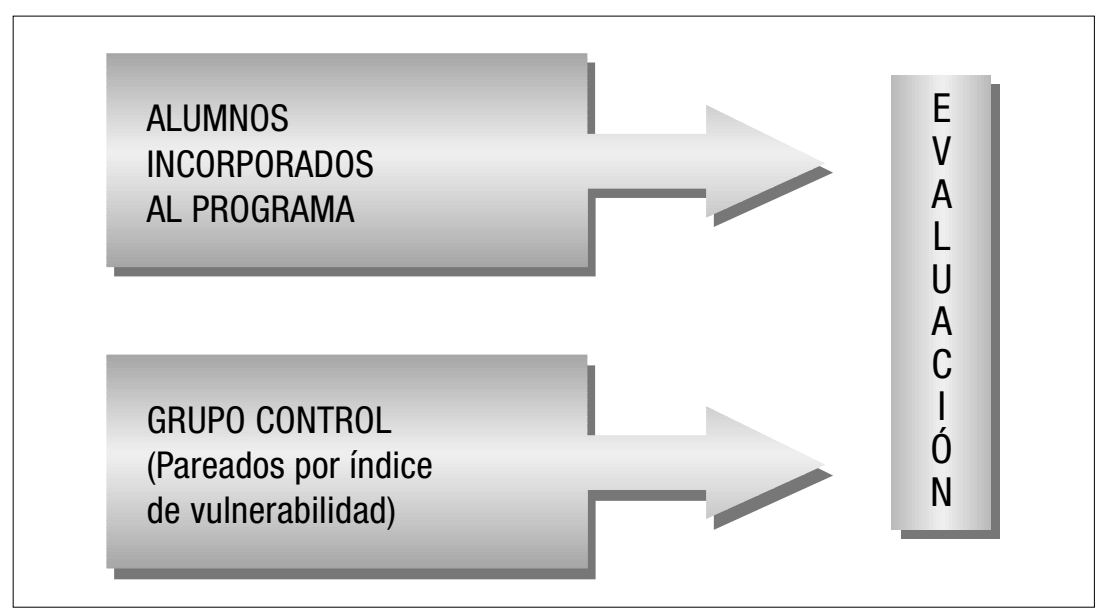




\section{DISEÑO DE LA INTERVENCIÓN}

Se diseñó una intervención sobre el grupo definido como vulnerable, en términos de aquellos alumnos que obtuvieron mayores puntajes en la evaluación de vulnerabilidad mediante la aplicación del índice presentado. Se optó por un diseño en modalidad de estudio con grupo control, para favorecer su evaluación. De este modo, se ofreció a los alumnos con mayores índices de vulnerabilidad una participación voluntaria en el programa, pareando posteriormente a los participantes, con un número equivalente de alumnos, con igual índice de vulnerabilidad, que se definió como grupo control.

Para los alumnos sujetos de la intervención, se ha programado un conjunto de actividades complementarias entre sí, las que comprenden dos actividades de taller y un programa de tutorías.

Los talleres diseñados se eligieron con base en un estudio de percepción de la salud mental en la Universidad Arturo Prat, realizado el año 2001. En él se comprobó que para los alumnos es relevante la necesidad de orientación y apoyo en áreas de desarrollo, relacionados con la etapa de la adolescencia, como los temas de sexualidad y pareja, grupos de pares, relaciones familiares y uso del tiempo libre. Todo ello se sistematizó en un taller de desarrollo personal en modalidad grupal, dirigido por una psicóloga clínica. Por otra parte, también se detectó la necesidad de apoyo específico en el área académica en torno a la adquisición de habilidades y destrezas necesarias para un rendimiento académico eficaz; en consecuencia, se diseñó un taller de hábitos y técnicas de estudio, también en modalidad grupal, dirigido por una psicóloga.

Como complemento de las actividades anteriores, se reconoció la conveniencia de desarrollar un programa de tutorías para estos alumnos, en el que estudiantes de cursos superiores de las mismas carreras, orientan y apoyan la integración y el desempeño académico de sus compañeros, supervisados por un equipo profesional del área de la salud mental, el que capacita además al equipo de tutores en el ejercicio de este rol. 


\section{Gráfico $\mathrm{N}^{\circ}$ 3:}

Se representan aquí las actividades del programa en las que el programa de tutorías acompaña a todo el proceso; los alumnos son incorporados además a dos talleres sucesivos, de Desarrollo Personal y de Técnicas y Hábitos de Estudio.

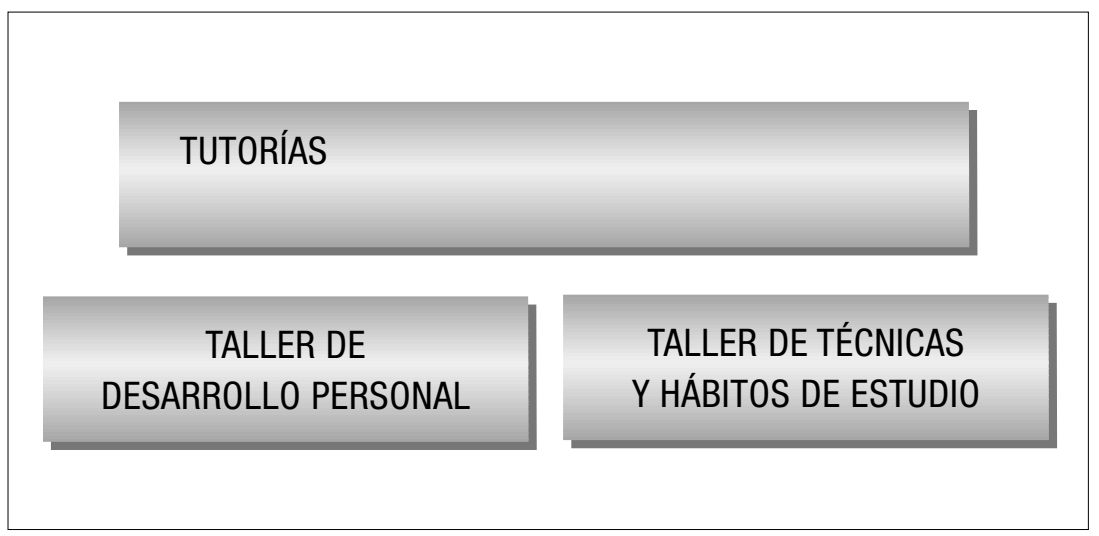

\section{CONTENIDOS DE LA INTERVENCIÓN}

\subsection{Sistema tutorías}

Las actividades de tutorías se sustentan en la convicción de que el apoyo entre pares permite importantes logros en diversos ámbitos; en el caso de los estudiantes universitarios, se considera no sólo su rendimiento académico, sino también su integración a la vida universitaria. Todo lo anterior se beneficia gracias a los proyectos, vivencias, experiencias y dificultades comunes que comparten los jóvenes compañeros de carrera al interior de la Universidad.

Se ha decidido la formación de un grupo de alumnos tutores, los que participarán en este sistema de trabajo de manera voluntaria, siempre que cumplan con algunos requisitos: estar cursando al menos el segundo año de su carrera, no enfrentar dificultades académicas serias y disponer de motivación y tiempo necesarios para el trabajo a realizar.

En consideración al grupo de alumnos de primer año incorporados al programa, el requerimiento de tutores se calcula en razón de un tutor por cada tres alumnos participantes de la misma 
carrera, con los que el tutor mantendrá un permanente contacto, asumiendo la responsabilidad de orientarlos en aspectos generales de la Universidad, apoyarlos en materias propias de estudio y favorecer su integración a la vida universitaria.

El trabajo de tutorías se inicia con una convocatoria a la inscripción de postulantes a tutores, los que posteriormente son seleccionados de acuerdo con sus antecedentes y con las necesidades del programa. Se considera una jornada de capacitación para los alumnos que ejercen el rol de tutorías, con la participación activa de estudiantes de la carrera de Sociología; en esta jornada, además de entregar contenidos específicos del programa, se busca recibir de estos jóvenes aportes y vivencias que permitan enriquecer las intervenciones.

\subsection{Taller de Técnicas y Hábitos de Estudio}

El Taller de Técnicas y Hábitos de Estudio responde a la necesidad detectada en los alumnos que ingresan a la Universidad, de contar con una instancia de apresto a las nuevas exigencias que implica la educación superior. En los alumnos de primer año se ha observado que un factor de vulnerabilidad específica es la carencia de un sistema de hábitos y técnicas de estudio acordes al ritmo universitario; éste se desarrolla en el curso del primer y segundo semestres, a partir del ensayo, del error y muchas veces a costa de la reprobación de asignaturas importantes.

El taller se propone acelerar y optimizar la adquisición de herramientas esenciales para el desempeño académico exitoso, proporcionando orientaciones prácticas para ser aplicadas en la toma de apuntes, preparación de pruebas, exámenes orales, disertaciones, etcétera.

El taller se ha diseñado para realizarse en sesiones modulares, para grupos de 10 participantes, con un total de cuatro sesiones en cada ciclo, con dos horas de duración cada sesión, y dirigidos por una psicóloga. Los contenidos se presentan en la siguiente tabla: 
TABLA N ${ }^{\circ}$ :

Contenidos del Taller de Técnicas y Hábitos de Estudio

\begin{tabular}{|l|l|l|}
\hline SESIÓN & \multicolumn{1}{|c|}{ TEMA } & \multicolumn{1}{c|}{ CONTENIDOS } \\
\hline PRIMERA & INTRODUCCIÓN AL TALLER & $\begin{array}{l}\text { Presentación } \\
\text { Definición de expectativas } \\
\text { Autodiagnóstico } \\
\text { Análisis de resultados } \\
\text { Contrato terapéutico }\end{array}$ \\
\hline SEGUNDA & ESTILOS DE APRENDIZAJE & $\begin{array}{l}\text { Toma de apuntes } \\
\text { Distribución del tiempo } \\
\text { (uso de agenda, tiempo libre, etcétera) }\end{array}$ \\
\hline TERCERA & TÉCNICAS DE ESTUDIO & $\begin{array}{l}\text { Preparación de pruebas } \\
\text { Resúmenes } \\
\text { Estudio grupal v/s individual } \\
\text { Memoria de corto y largo plazo }\end{array}$ \\
\hline CUARTA & MANEJO DE LA ANSIEDAD & $\begin{array}{l}\text { Reconocimiento de síntomas } \\
\text { de ansiedad } \\
\text { Técnicas de autocontrol y relajación } \\
\text { Disertación y exámenes orales } \\
\text { Niveles adaptativos y tensión }\end{array}$ \\
& & Evaluación y cierre del taller \\
\hline
\end{tabular}

\subsection{Taller de Desarrollo Personal}

Podemos concebir el desarrollo personal como la actualización de las potencialidades afectivas, cognitivas y sociales del individuo. Estas dimensiones no se encuentran separadas unas de las otras, sino que se superponen entre sí; de esta forma, la integración del mundo afectivo con la esfera intelectual y ambas con la esfera social definen un desarrollo y crecimiento armónico y saludable en cada individuo, que fortalece así la adaptación humana, es decir, la capacidad de hacer frente de manera satisfactoria a las innumerables tareas de cada etapa de la existencia.

Una de las tareas relevantes y decisivas para la vida es la elección de una carrera o profesión, que determinará ese aspecto preponderante de la existencia de cada persona, que es el ámbito laboral en el que el individuo se podrá desempeñar.

Por lo anterior, hemos considerado necesario incluir en la formación profesional de los estudiantes de la Universidad Arturo Prat 
un Programa de Desarrollo Personal, que prepare y favorezca la adaptación a este período de formación académica.

\subsubsection{Objetivo general}

Al final del taller los alumnos habrán internalizado conceptos y vivencias que favorecerán una mayor adaptación en su quehacer social, cognitivo y afectivo.

\subsubsection{Metodología}

Se utilizará el sistema de "taller" semiestructurado, que contará con 6 sesiones de 2 horas cada una, con una frecuencia de una vez por semana. Cada sesión tendrá una parte expositiva, en la que se entregará y discutirá una base conceptual apropiada a cada tema, y una parte experiencial, donde se vivenciarán los temas planificados para cada sesión, utilizando en ambos técnicas como el trabajo en parejas, pequeños grupos, ejercicios de relajación, imaginería u otros.

\subsubsection{Contenido de las sesiones}

- $1^{\circ}$ sesión: Paradigmas y estilo atribucional. Autoconcepto.

- $2^{a}$ sesión: Estilo cognitivo-afectivo: ABC de Ellis. Autoestima.

- $3^{a}$ sesión: Conciencia del esquema corporal. Autocuidado.

- $4^{a}$ sesión: Estrategia de solución de problemas.

- $5^{a}$ sesión: Comunicación y empatía. Asertividad.

- $6^{a}$ sesión: Relaciones interpersonales. Sentido de vida.

\section{RESULTADOS ESPERADOS Y EVALUACIÓN}

Se trata de un programa que al momento de esta presentación, en septiembre de 2002, se encuentra en plena ejecución. Por lo tanto, sólo cabe destacar en el presente artículo que lo que se busca con este trabajo es favorecer la equidad en el quehacer universitario, como herramienta de retención y promoción del éxito académico entre los alumnos de primer año. Los resultados que esperamos dicen relación con lograr una efectiva protección de los alumnos definidos como más vulnerables, medidos en mejores rendimientos académicos y mayor satisfacción en la integración de los estudiantes a la vida universitaria. 
Por consiguiente, la evaluación de resultados no sólo deberá incluir los datos objetivos de rendimiento, sino también el grado de integración y satisfacción que logren los alumnos cubiertos con el programa, en comparación con sus compañeros no incorporados (grupo control). Con todo, consideramos la instancia de evaluación un espacio que, en la medida en que se pueda hacer en forma amplia y participativa, también permitirá estimular la reflexión de la comunidad universitaria sobre el tema de construir equidad en nuestro sistema educacional. 


\section{ReFERENCIAS BibLIOGRÁFICAS y NOTAS}

Anderson, L.E., Social Mobility in Latin America, Instituto de Investigaciones Sociales y Económicas, Universidad Católica Boliviana; documento de trabajo Nº3/2000, Bolivia, 2001.

Anderson, L. E., Baja movilidad social en Bolivia: causas y consecuencias para el desarrollo, Instituto de Investigaciones Sociales y Económicas, Universidad Católica Boliviana, Bolivia, 2002, en http:/ Ins.ucb.edu.bo./

Ellis, A. y Dryden, W., Práctica de la terapia racional emotiva, Desclée de Brouwer, Bilbao, España, 1989.

Frankl, Victor, El hombre en busca de su sentido, Herder, Barcelona, España, 1996.

Hassler, J. y Rodríguez Mora, J., "Social Mobility and Growth"; en Seminar Paper $N^{\circ}$ 635, Institute for International Economics Studies, Stockholm University, Suecia, 1998.

Varios, Chile en Cifras, Estadísticas de Población, Instituto Nacional de Estadísticas, Santiago, Chile, 2002, en http://www.ine.cl

Osorio, Domínguez, Valdivia, Iglesias, Miquel y Gómez, Percepción de la situación de salud mental; Universidad Arturo Prat, Ediciones Campus, Iquique, Chile, 2001.

Maslow, Abraham, El hombre autorrealizado, Kairós, Barcelona, España, 1998.

Maturana, Humberto, Fundamentos biológicos de la realidad. La realidad ¿Objetiva o construida?, T. 1, Anthropos, Barcelona, España, 1997.

Compendio de educación superior, Ministerio de Educación, Santiago, Chile, 2002, en http://www.mineduc.cl/superior/compendio 
Murphy, K.M., Shleifer, A., Vishny, R.W., "The Allocation of Talent: Implications for Growth"; en Quarterly Journal of Economics, Vol 106(2), M I T Press, Massachusetts, EE.UU., 1991 , http:// mitpress.mit.edu.journals/.

Watslavick, Paul et al, Teoría de la Comunicación Humana, Herder, Barcelona, España, 1982. 